\title{
TJ-II operation tracking from Cadarache
}

J. Vega, E. Sánchez, A. Portas, A. Pereira, A. López, E. Ascasíbar

Asociación EURATOM/CIEMAT para Fusión, Madrid, Spain

S. Balme, Y. Buravand, P. Lebourg, J. M. Theis, N. Utzel

Association EURATOM/CEA sur la Fusion, Cadarache, France

M. Ruiz, E. Barrera, S. López, D. Machón

Dpto. Sistemas Electrónicos y de Control, UPM, Madrid, Spain

R. Castro, D. López

Red.es - RedIRIS. Madrid. Spain

A. Mollinedo, J. A. Muñoz

CIEMAT. Computing Center. Madrid. Spain

\begin{abstract}
The TJ-II remote participation system was designed to follow the TJ-II discharge production, even allowing the physicist in charge of operation to be in a remote location. The system has been based on both web servers and Java technology. These elements were chosen due to its open character, security properties, platform independence and technological maturity. Web pages and Java applications permit users to access experimental systems, data servers and the operation logbook. Security resources are provided by the PAPI system, a distributed authentication and authorization system.

The TJ-II remote participation tools have allowed us to command and follow the stellarator operation from Cadarache. Over 1,000 digitizer channels and more than 20 diagnostic control systems were remotely available from web pages for monitoring/programming purposes. One Java application provided on-line information about the acquisition status of channels and acquisition cards. A second Java application showed temporal evolution signals that were refreshed in an automated way on the screen after each shot. A third Java application provided access to the operation logbook. In addition to these tools, we used the VRVS videoconferencing system (FUSION community, X-Point room) and the EFDA Messenger Service for instant messaging (Jabber client).
\end{abstract}

\section{INTRODUCTION}

Nowadays, remote access to fusion devices has become a crucial point. Remote participation methods must provide participants with tools to compensate for the lack of direct personal interaction. The tools can be classified in two different groups. First, we have the set of means related to audio-visual media, i.e. collaborative tools for videoconference, instant messaging and shared displays in order to follow meetings and seminars as well as participating in the control room atmosphere. Second, people need resources for the interplay with experimental systems, computers and databases. Typical capabilities of this group allow people the monitoring/programming of experimental 
systems (data acquisition and diagnostic control systems), data visualization, data integration/retrieval, operation logbook reading/writing and realtime operation tracking. Of course, these tools depend exclusively upon the experimental environment of the fusion device and, therefore, they are generated necessarily from ad-hoc developments: digitizer programming software, data access libraries, interaction with diagnostic control systems and logbook software.

This article describes the remote command of the TJ-II fusion device from Cadarache. TJ-II is a medium size stellarator (heliac type) [1] located at CIEMAT in Madrid (Spain). It is a four period device whose main parameters are: $\mathrm{B}(0)=1.2 \mathrm{~T}$, $\mathrm{R}(0)=1.5 \mathrm{~m},\langle\mathrm{a}\rangle=0.22 \mathrm{~m}$. Two gyrotrons $\left(300 \mathrm{~kW}\right.$ each, $53.2 \mathrm{GHz}, 2^{\text {nd }}$ harmonic, $\mathrm{X}-$ mode polarization) and one NBI $(300 \mathrm{~kW})$ provide plasma heating. Section 2 summarizes the TJ-II operation environment. Section 3 explains the software tools to follow the TJ-II operation from Cadarache. Section 4 describes the operation, related problems and their later solutions. Finally, section 5 shows some conclusions.

\section{TJ-II OPERATION ENVIRONMENT}

The TJ-II operation environment is made up of a set of resources which provide service not only to local area network (LAN) users but also to external users [2]. Local and remote participants use exactly the same software tools to supervise and configure experimental systems and to access the operation logbook [3]. The operation environment has been based on web servers and Java technologies because both of them allow the creation of the most standard operation environment not depending on computers, operating systems, software licenses or hardware architectures. Moreover, web technologies let us easily organize a very scalable system architecture not only depending on one web server, but on several ones.

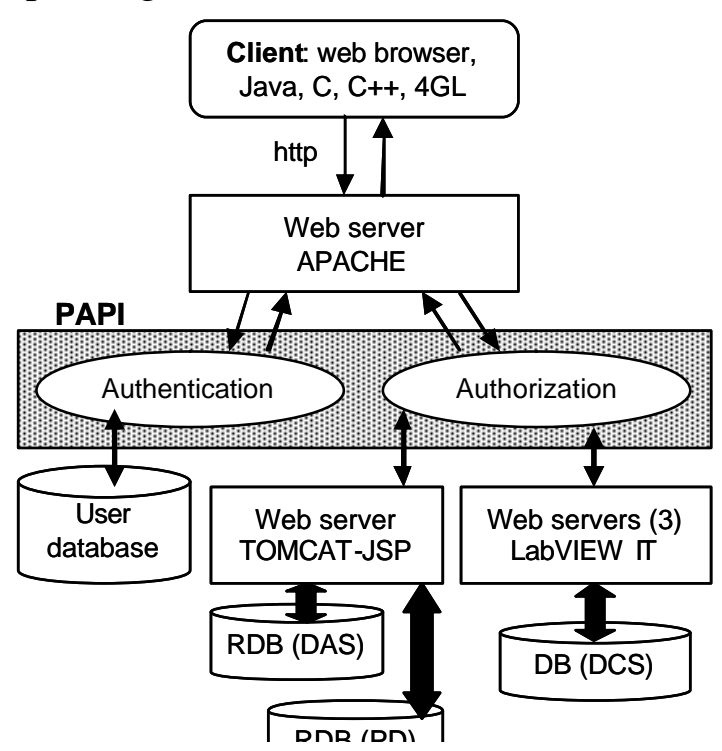

Fig. 1. Relational databases (RDB) manage all the information related to data acquisition systems (DAS), diagnostic control systems (DCS) and phvsical data $(P D)$
Network services are provided through resources supplied by Java Server Pages (JSP pages). The client part makes use of web browsers and ad-hoc Java applications. All software running in the TJ-II operation environment was developed following a three tier model [4], in which there is one tier devoted to providing graphic user interface only. Java applications deployment is achieved by means of the Java Network Launch Protocol (JNLP) technology, in particular the Java Web Start application [5].

Access security is based on a distributed authentication and authorization system. The characteristic of 'distributed' was a basic requirement taking into account the distributed nature of web technologies. The PAPI system is used here [6].

Figure 1 sums up the TJ-II operation environment architecture [3]. A client can be a web browser or any executable application (Java, C, C++, LabView or script files). Currently, the TJ-II experimental environment uses four web servers, one dedicated to 
operation tracking and data acquisition tasks, the others assigned to the diagnostic control systems [7].

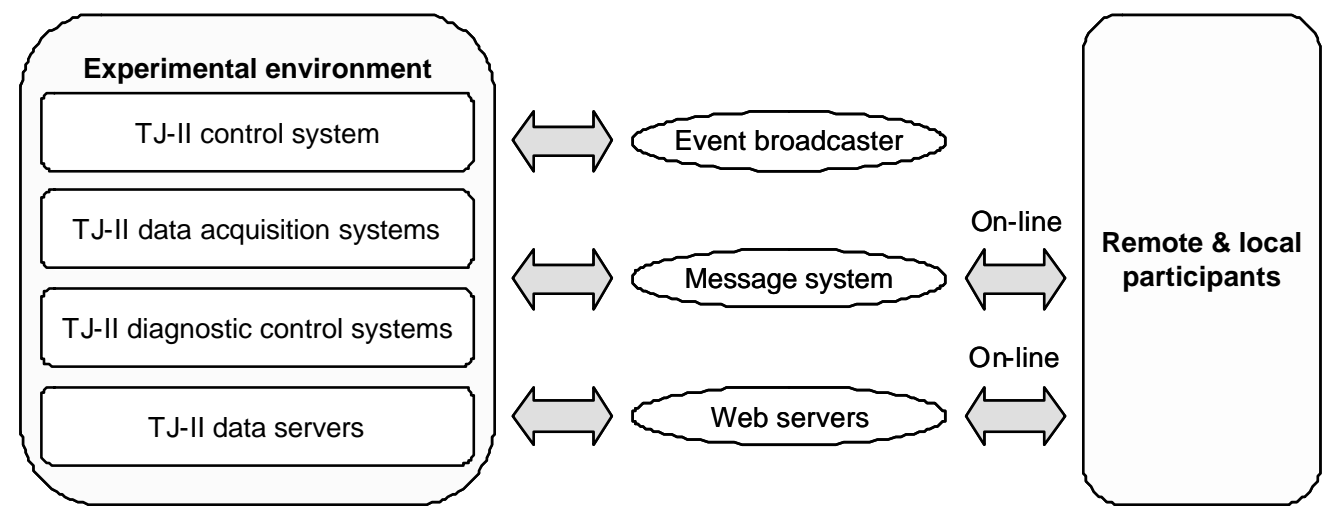

Fig. 2. Communication flows during TJ-II operation. Users make use of on-line communication capabilities through web servers (synchronous) and a message system (asynchronous)

Figure 2 summarizes communication flows during TJ-II operation. The complete experimental environment is on the left, the communication mechanisms are in the middle, and the users (local or remote) on the right. There is an event broadcaster on top. It is a software development for synchronizing software programs with the TJ-II operation [8, 9]. The event broadcaster is based on the Berkeley sockets application program interface (API) and, therefore, it is only used within the local area network to prevent establishing rules in the firewall. Web servers are at the bottom. They are devoted to on-line synchronous communications between users and the TJ-II environment. Finally, there is a message system. It is a message oriented middleware based on a Java API and it allows on-line asynchronous communications between both ends [10]. It is used to broadcast operation messages in order to maintain and distribute on- line information about TJ-II operation.

\section{TJ-II REMOTE OPERATION FROM CADARACHE}

The aim of the TJ-II remote operation was to demonstrate the validity of the TJII remote participation model that is based on web servers and Java technologies. These elements allow the configuration of a real multiplatform environment for participants. The environment depends only on free typical computer components: web browsers, Java Virtual Machines and deployment strategies based on the JNLP protocol and Web Start technologies.

The TJ-II operation was commanded from a videoconference room in Tore Supra site. Two personal computers (Windows XP based) were used. The only installations required in the computers were the upgrade of the Java Virtual Machines and the download of the Java Web Start application (http://java.sun.com/products/javawebstart). The VRVS videoconference system was used between the videoconference room in Cadarache and the TJ-II control room in Madrid. We connected to the X-Point virtual room of the FUSION community. Also, instant messaging was used. Connections to the main page of the TJ-II remote participation system (http://tj2web.ciemat.es) were carried out with Internet Explorer V6.

TJ-II web pages provided access to all resources: over 1000 digitization channels, 20 diagnostic control systems, operation logbook, data acquisition systems 
monitoring and signals visualization. In addition, we tried to control and monitor a continuous data analysis.

\subsection{Digitization channels and diagnostic control systems}

A set of web pages allow users to access the acquisition set-up of the digitizers. Users only need to access the list of signals and their descriptions in order to participate efficiently in TJ-II experiments (knowledge of signal hardware allocation is not needed [4]). Read/write permissions of users are managed by PAPI.

Each diagnostic control system has its own web page. The page refreshes its content on a periodical basis (user defined) to show all operation parameters. Moreover, only authorized users can modify the settings.

\subsection{Operation logbook}

Users have full access in read only mode to the web pages which provide the operation logbook information. Users can access the information by means of relational searches. Only the physicist in charge of operation has writing permissions. Data input is accomplished by means of a Java application which can be downloaded from a web page. The Java Web Start application verifies in an automated way the existence of a new version of the operation logbook each time the Java application is executed. If there is a new version, it is downloaded from the web server and then it is executed. It should be noted that the possibility of software download does not ensure the unlimited execution of the software. The PAPI system always verifies execution permissions when an application starts.

\subsection{Data acquisition systems monitoring}

It is a Java application whose graphical user interface provides a constant update of the status of any data acquisition system. This status represents the situation of its signals. Possible signal situations include READY (waiting for a new discharge), ARMED (waiting for a trigger to begin digitalisation) and SENDING (data integration into the database). Status changes are asynchronous ly notified by means of the message middleware. Software update is performed in an automated way when the application starts and a new software version is available.

\subsection{Signal visualization}

It is another Java application, also with automated software distribution, that displays a set of pre-programmed monitor signals. It was a very simple version which showed, without user intervention, experimental signals as discharges took place. The asynchronous message middleware was in charge of data sending.

\subsection{Remote interaction with continuous data analysis}

This part was designed to evaluate latencies between commands issued remotely and its execution with 'hot swap' of software modules. We used a multiprocessor architecture based on a PXI system [11] as a target system. The main controller (Linux based) was in charge of data acquisition and remote input commands. A processing card (Linux based) was programmed to perform three different kinds of processes: simple 
signal visualization as the waveform is digitized, a continuously computing of the signal fast Fourier transform and its visualization, and signal modulation in amplitude and its visualization. A digitizer card received a continuous input from a waveform generator. We programmed a sinusoidal signal. Data acquisition and analysis programs were written in LabVIEW.

Remote user interaction was carried out through a web page that provided controls to select the type of process to perform and also a VNC screen to show the console of the processing card with the visualization required. The message system was in charge of asynchronous notifications to the PXI main controller about user process selection. Then, the main controller transmitted the order to the processing card that performed the 'hot swap' of software modules according to the user requirement.

\section{DISCUSSION}

The connection with Madrid was started successfully with the VRVS videoconferencing system. One PC was used by the physicist in charge (PIC) of operation to command the discharges and other PC was used as simple user terminal. The accesses through the web pages to data acquisition systems $(1000+$ digitization channels), diagnostic control systems, PIC comments and configurations about rext discharge and relational searches on logbook data were also successfully. The PIC downloaded and executed the Java application corresponding to the logbook. Information was recorded after each shot without problems.

However, Java applications connected with both the monitoring of data acquisition systems and signal visualization were apparently downloaded, but the execution did not begin. Finally, the web page with the VNC screen did not respond to user selections.

Therefore, information access through web servers worked perfectly but troubles appeared when the message system was used. We realized that in these cases, a TCP/IP port (about port 5000) was open in the PC waiting for data from the message system. The only explanation to this behaviour was that the client API of the message system created the ports. The Tore-Supra local area networks have a very restrictive access method and their firewalls only enable input traffic to well-known ports. Consequently, all traffic from the asynchronous message system was being filtered and the applications remained in a 'hang' state.

To verify this hypothesis, we connected to the TJ-II environment by means of a telephone line and a free Internet Service Provider (ISP), instead of the Tore-Supra LAN. Of course, bandwidth was greatly reduced, but we were able to download and execute the above Java applications. Channel status changes and signal updates allowed us to have on-line information about TJ-II experimental systems and signals. It should be remarked that this is the normal behaviour from our users in Spanish Universities because their access methods to their LAN environments are not as limiting as in Tore Supra.

Nevertheless, the remote interaction with the continuous data analysis seemed not to respond properly to user selections. In fact, we were able to select remotely Fourier analysis visualization only once.

Hence, two different proble ms were detected. First, we were working with a bad configuration of the message system: http tunnelling was not in operation. Second, we had a synchronization problem with running tasks between the PXI main controller and the processing card. 
Both problems were perfectly identified but they could not be solved from Cadarache because the solutions implied major amendments in software codes and configuration properties. Regarding the message system, a first and fast approach to the solution was to modify the communication flows. Each remote application, instead of communicating with the message system, had to establish communication with a JSP page and this page made use of the message system from the TJ-II local area network. In this way, remote applications were connected to JSP pages through web servers and message system connections took place within a local area network environment. We tested this solution and we succeeded. After this, a full message system with http tunnelling was put into operation and, presently, it is working [10].

Concerning the second problem, we fixed a bug linked to task synchronization and now, the proposed test works.

\section{CONCLUSIONS}

The PAPI system managed very efficiently and very transparently all authentication and authorization requirements. The interplay existing between users and the remote participation environment through web servers did not show any problem at all. Message systems for remote participation must be built with http tunnelling properties to avoid packet filtering by the firewalls. Java application deployment based on JNLP and Web Start provides a reliable way of automatic software distribution. We use the asynchronous message system to distribute to all client applications the temporal evolution signals of discharges. However, a better approach to save resources in the message system would consist of distributing the availability of the signals and, then, the applications themselves ask for the signals to the web servers.

\section{ACKNOWLEDGMENTS}

This work is partially funded by the Spanish Ministry of Education and Science under the Project No. ENE2004-07335.

\section{REFERENCES}

[1] C. Alejaldre et al. Plasma Phys. Controlled Fusion 41, 1 (1999), pp. A539.

[2] J. Vega et al. Rev. Sci. Instr. 74, 3 (2003) 1773-1777.

[3] J. Vega et al. Fus. Eng. Des. In press.

[4] J. Vega et al. Rev. Sci. Instr. 75, 10 (2004) 4251-4253.

[5] M. Marinilli. Java Deployment with JNLP and Web Start. Sams Publishing (2001).

[6] R. Castro-Rojo et al. Computer Networks 37, 6 (2001) 703-710.

[7] A. López et al. Fus. Eng. Des. 60 (2002) 487-492.

[8] J. Vega et al. Fus. Eng. Des. 71 (2004) 117-121.

[9] A. Pereira et al. Sent to Fus. Eng. Des.

[10] E. Sánchez et al. Sent to Fus. Eng. Des.

[11] M. Ruiz et al. Rev. Sci. Ins. 75, 10 (2004) 4261-4264 